\title{
The Riemann-Hilbert Formalism For Certain Linear and Nonlinear Integrable PDEs
}

\author{
Dimitrios A PINOTSIS ${ }^{a}$ \\ ${ }^{a}$ Department of Mathematics \\ University of Reading \\ Reading, RG6 6AX, UK \\ E-mail:d.pinotsis@reading.ac.uk
}

Received March 2, 2007; Accepted in April 26, 2007

\begin{abstract}
We show that by deforming the Riemann-Hilbert (RH) formalism associated with certain linear PDEs and using the so-called dressing method, it is possible to derive in an algorithmic way nonlinear integrable versions of these equations.

In the usual Dressing Method, one first postulates a matrix RH problem and then constructs dressing operators. Here we present an algorithmic construction of matrix Riemann-Hilbert $(\mathrm{RH})$ problems appropriate for the dressing method as opposed to postulating them ad hoc. Furthermore, we introduce two mechanisms for the construction of the relevant dressing operators: The first uses operators with the same dispersive part, but with different decay at infinity, while the second uses pairs of operators corresponding to different Lax pairs of the same linear equation. As an application of our approach, we derive the NLS, derivative NLS, KdV, modified KdV and sine-Gordon equations.
\end{abstract}

\section{Introduction}

We present an algorithmic approach for the derivation of nonlinear integrable equations in 1+1 dimensions. Namely, we show that a systematic deformation of the Riemann-Hilbert $(\mathrm{RH})$ problems associated with certain linear equations yields matrix $\mathrm{RH}$ problems which, using the the so-called dressing method, lead to nonlinear integrable versions of these linear PDEs. The RH problems associated with linear equations are obtained by performing the spectral analysis of a pair of linear eigenvalue equations.

Our approach consists of the following steps: (i) Given a linear PDE we use the formulation of [5] to construct a Lax pair of this PDE, i.e., a pair of compatible linear eigenvalue equations. For example, let $q(x, t)$ satisfy the Stokes equation,

$$
q_{t}+q_{x x x}=0 .
$$

It was shown in [5] that this equation can be written as the compatibility condition of the following pair of linear eigenvalue equations involving the scalar function $\mu(x, t, k)$ : 


$$
\mu_{x}+i k \mu=q, \mu_{t}+i k^{3} \mu=-q_{x x}+i k q_{x}+k^{2} q .
$$

(ii) Use this pair to derive a matrix $\mathrm{RH}$ problem associated with the linear equation. The spectral analysis of (1.2) yields the following scalar $\mathrm{RH}$ problem

$$
\mu^{+}(x, t, k)-\mu^{-}(x, t, k)=e^{i k x+i k^{3} t} \rho_{0}(k), \quad k \in \mathbb{R}, \quad \mu=O\left(\frac{1}{k}\right),
$$

where $\rho_{0}$ is some function of $k$ with appropriate smoothness and decay and $\mu^{+}, \mu^{-}$are the limits of the function $\mu(x, t, k)$ as $k$ approaches the real axis.

Let $Z$ be a $2 x 2$ matrix

$$
Z=\left(\begin{array}{ll}
Z_{11} & Z_{12} \\
Z_{21} & Z_{22}
\end{array}\right)
$$

Then, equation (1.3) can be rewritten in the following matrix form:

$$
\begin{aligned}
& Z^{+}=Z^{-} S_{l}(x, t, k), \quad k \in \mathbb{R}, \\
& Z \rightarrow I_{2}+O\left(\frac{1}{k}\right), \quad k \rightarrow \infty,
\end{aligned}
$$

where $Z^{+}(x, t, k)$ and $Z^{-}(x, t, k)$ are matrix functions which are analytic in the upper and lower half complex $k$-plane respectively. Also, the "jump " matrix $S_{l}(x, t, k)$ is defined by

$$
S_{l}(x, t, k)=\left(\begin{array}{ll}
1 & e^{i k x+i k^{3} t} \rho_{0}(k) \\
0 & 1
\end{array}\right) .
$$

We note that $S_{l}$ is triangular and satisfies $\operatorname{det} S_{l}(x, t, k)=1$. The triangular form of this matrix reflects the scalar nature of the relevant $\mathrm{RH}$ problem, while the unimodularity is necessary for the unique solvability of this problem.

(iii) Deform this $\mathrm{RH}$ problem to obtain a new matrix $\mathrm{RH}$ problem which is appropriate for the dressing method and apply this method. A RH problem is appropriate for the dressing method when the relevant jump matrix is non-triangular and unimodular.

In the following, we propose a systematic deformation of the RH problem (1.4)-(1.5). Namely, equation (1.1) in addition to (1.2), has also the Lax pair given by the equations resulting from (1.2) after replacing $k$ with $-k$. By performing the spectral analysis of this Lax pair we obtain an equation of the form (1.4) with $S_{l}(x, t, k)$ replaced by $S_{l}^{T}(x, t,-k)$. Using this $\mathrm{RH}$ problem and the $\mathrm{RH}$ problem (1.4)-(1.5) it is possible to construct $\mathrm{RH}$ problems which can be associated with nonlinear versions of (1.1). Indeed, starting from a $\mathrm{RH}$ problem of the form (1.4)-(1.5) where the matrix $S_{l}(x, t, k)$ is replaced by

$$
S(x, t, k)=S_{l}^{T}(x, t,-k) S_{l}(x, t, k),
$$

and using the dressing method we obtain the following non-Abelian version of (1.2)

$$
Z_{x}+i k\left[\sigma_{3}, Z\right]=Q Z, \quad Z_{t}+4 i k^{3}\left[\sigma_{3}, Z\right]=H Z,
$$


where $\sigma_{3}$ denotes the third Pauli matrix,

$$
\sigma_{3}=\left(\begin{array}{ll}
1 & 0 \\
0 & -1
\end{array}\right)
$$

and the matrices $Q, H$ appearing in the right hand side of the above equations are of certain order in $k$ and involve the function $q$ and its derivatives.

Furthermore, we introduce two mechanisms for the construction of dressing operators. These mechanisms yield nonlinear equations possessing the same linear part, but with different nonlinear terms:

(i) We consider pairs of dressing operators with different decays at infinity. In particular, for the case of equation (1.1), we consider the following operators

$$
\partial_{x}+i k \widehat{\sigma_{3}}-Q, \quad \partial_{t}+4 i k^{3} \widehat{\sigma_{3}}-H,
$$

where $\widehat{\sigma}_{3}$ denotes the matrix commutator with $\sigma_{3}$

$$
\widehat{\sigma}_{3} Z=\left[\sigma_{3}, Z\right],
$$

and $Z$ is a $2 x 2$ matrix. If $Q(k)$ is of order $O(1)$ and $H(k)$ is of order $O\left(k^{2}\right)$ the compatibility of equations (1.8) yields the modified $\mathrm{KdV}$ equation

$$
q_{t}+q_{x x x}+6 q^{2} q_{x}=0,
$$

while, if $Q(k)$ is of order $O\left(\frac{1}{k}\right)$ and $H(k)$ is of order $O(k)$ we find the well-known $\mathrm{KdV}$ equation

$$
q+q_{x x x}+6 q q_{x}=0 .
$$

(ii) We consider dressing operators involving different powers of $k$. These operators correspond to different Lax pairs of the same linear equation. For example, for the linearised Schroedinger equation

$$
i q_{t}+q_{x x}=0,
$$

one pair of associated dressing operators is given by

$$
\partial_{x}+i k \widehat{\sigma}_{3}, \quad \partial_{t}+2 i k^{2} \widehat{\sigma}_{3} .
$$

The compatibility of the relevant Lax pair yields the Nonlinear Schroedinger (NLS) equation

$$
i q_{t}+q_{x x}-2 \lambda|q|^{2} q=0, \quad \lambda= \pm 1 .
$$

Similarly, using the dressing operators which are obtained from (1.14) after letting $k \rightarrow k^{2}$ we find the derivative NLS equation

$$
i q_{t}+q_{x x}-\lambda\left(|q|^{2} q\right)_{x}=0, \quad \lambda= \pm 1 .
$$

\section{Assumptions}

We assume that the initial conditions belong to the class of Schwartz functions denoted by $S(R)$. 


\section{Lax pairs and the RH formalism for Linear Equations}

A large class of linear and nonlinear integrable PDEs, including linear PDEs with constant coefficients, can be written as the compatibility condition of two linear ordinary differential equations (ODEs), the so-called Lax pair. It should be noted that Lax pairs of linear equations, in contrast to Lax pairs of the nonlinear equations, can be obtained algorithmically. In particular, it was shown in [5] that every linear PDE with constant coefficients in two dimensions

$$
P\left(\partial_{x}, \partial_{t}\right) q=0
$$

possesses the Lax pair:

$$
\begin{aligned}
& \mu_{x}+i k \mu=q, \quad k \in C, \\
& P\left(\partial_{x}, \partial_{t}\right) \mu=0,
\end{aligned}
$$

where $\mu(x, t, k)$ is a scalar function. For example, using a variation of the above result we find that the sine-Gordon equation

$$
q_{x t}+q=0,
$$

can be written as the compatibility condition of equation (2.2) with $q$ replaced by $q_{x}$ and of the following equation

$$
\mu_{t}+\frac{i}{k} \mu=\frac{i}{k} q .
$$

Indeed, equation (2.3) yields

$$
\mu_{x t}+\mu=0 .
$$

Taking the derivative with respect to $t$ of $(2.2)$ where $q$ is replaced by $q_{x}$ and then substituting the above equation in the resulting equation, we obtain (2.5).

For linear evolution equations, the Lax pair involves an ordinary differential equation (ODE) in $x$, namely equation (2.2), as well as an ODE in $t$. Following [5], we derive two families of Lax pairs for dispersive evolution equations. Indeed, let $q(x, t)$ satisfy a general dispersive evolution equation of order $n$,

$$
\left(\partial_{t}+i w\left(-i \partial_{x}\right)\right) q(x, t)=0,
$$

where $w(k)$ is a polynomial of order $n$,

$$
w(k)=\alpha_{0}+\alpha_{1} k+\cdots+\alpha_{n} k^{n}, n \in N^{+},
$$

where $\left\{a_{j}\right\}_{1}^{n}$ real. Then, this equation possesses the Lax pairs

(i)

$$
\mu_{x}+i k \mu=q(x, t), \quad \mu_{t}+i w(k) \mu=-X(x, t, k)
$$


where

$$
X(x, t, k)=-\left(\frac{w(k)-w(l)}{k+l}\right) q,
$$

(ii)

$$
\mu_{x}-i k \mu=q(x, t), \quad \mu_{t}+i w(k) \mu=-Y(x, t, k)
$$

where

$$
Y(x, t, k)=\left(\frac{w(k)-w(l)}{k-l}\right) q,
$$

and $l \doteqdot-i \partial_{x}$.

Equations (2.9) and (2.11) with $k$ replaced by $k^{n}$, where $n$ is a positive integer, are also Lax pairs of equation (2.7). Therefore, equation (2.7) possesses two families of Lax pairs given by (2.9) and (2.11) where $k$ is replaced by $k^{n}$. Each value of $n$ gives a particular member of these families.

In [7], the Lax pair associated with (1.13) was used to solve the Cauchy problem on the infinite line. In the process, a scalar $\mathrm{RH}$ problem associated with (1.13) was obtained. Here, using the Lax pair formulation of linear PDEs, we derive in an algorithmic way matrix Riemann-Hilbert problems associated with linear equations. As illustrative examples, we derive Riemann-Hilbert problems associated with two equations of the form (2.7), namely equations (1.1) and (1.13). We also show that a similar approach is possible for other linear equations, which are not of the form (2.7), such as (2.4).

Proposition 2.1. Let $Z(x, t, k)$ satisfy the matrix $R H$ problem (1.4)-(1.5) where the "jump " matrix $S_{l}(x, t, k)$ is given by

$$
S_{l}(x, t, k)=\left(\begin{array}{ll}
1 & e^{-i \phi(x, t, \kappa)} \rho_{0}(k) \\
0 & 1
\end{array}\right)
$$

with

(i) $\phi(x, t, k)=k x+k^{2} t$

(ii) $\phi(x, t, k)=k x+k^{3} t$,

(iii) $\phi(x, t, k)=k x+\frac{1}{k} t$.

Let $\rho_{0}(k)$ be given by

(i)-(ii)

$$
\rho_{0}(k)=\int_{-\infty}^{\infty} e^{i k x} q_{0}(x) d x,
$$

(iii)

$$
\rho_{0}(k)=\int_{-\infty}^{\infty} e^{i k x} q_{1}(x) d x .
$$

Then $Z_{12}$ is given by

$$
Z_{12}(x, t, k)=\frac{1}{2 \pi i} \int_{-\infty}^{\infty} \frac{e^{-i \phi(x, t, \kappa)} \rho_{0}(\kappa)}{\kappa-k} d \kappa .
$$


Also, the complex-valued function $q(x, t)$ satisfies

(i) the linearised Schroedinger equation (1.13) with $q(x, 0)=q_{0}(x) \in S(R)$,

(ii) the Stokes equation (1.1) with $q(x, 0)=q_{0}(x) \in S(R)$,

(iii) the sine-Gordon equation (2.4) with $q(x, 0)=q_{0}(x), q_{x}(x, 0)=q_{1}(x)$ and $q_{0}(x)-$ $2 \pi m, q_{1}(x) \in S(R)$ where $m$ is an integer and $x \in(-\infty, \infty), t \in[0, \infty)$.

Proof.

Equations (1.4)-(1.5) are equivalent to

$$
\begin{aligned}
Z_{11}^{+} & =Z_{11}^{-}, \quad Z_{21}^{+}=Z_{21}^{-}, \quad k \in \mathbb{R} \\
Z_{12}^{+}-Z_{12}^{-} & =e^{-(i k x+i w(k) t)} \rho_{0}(k) Z_{11}^{-}, Z_{22}^{+}-Z_{22}^{-}=e^{-(i k x+i w(k) t)} \rho_{0}(k) Z_{21}^{-}(2.1 \\
Z_{11}^{ \pm}, Z_{22}^{ \pm} \rightarrow 1, & Z_{12}^{ \pm}, Z_{21}^{ \pm} \rightarrow O\left(\frac{1}{k}\right) .
\end{aligned}
$$

The above jump and boundary conditions yield $Z_{11}=Z_{22}=1$ and $Z_{21}=0$. Furthermore, equation (2.18a) and the Plemelj formulae [2] imply that $Z_{12}$ is given by (2.16).

The spectral analysis of (2.9) yields the $\mathrm{RH}$ problem

$$
\mu^{+}(x, t, k)-\mu^{-}(x, t, k)=e^{-(i k x+i w(k) t)} \rho_{0}(k), \quad k \in \mathbb{R}, \quad \mu=O\left(\frac{1}{k}\right) .
$$

This $\mathrm{RH}$ problem is identical with the $\mathrm{RH}$ problem satisfied by $Z_{12}$, namely equation (2.18a). Therefore, $Z_{12}=\mu$ and the result follows from the definition of Lax pairs.

QED

\section{Non-Abelian Lax Pairs}

The spectral analysis of (2.11) yields a RH problem of the form (1.4)-(1.5) where the jump matrix is obtained from (2.13) after letting $k \rightarrow-k$. It turns out that one can use a combination of the matrices appearing in this $\mathrm{RH}$ problem and in the $\mathrm{RH}$ of Proposition 2.1 for the derivation of nonlinear versions of (2.7). Namely, the requirements of unimodularity and non-triangularity imply that $S_{l}(k)$ should be replaced by one of the following: $S_{l}^{T}(-k) S_{l}(k), S_{l}(k) S_{l}^{T}(-k), S_{l}^{T}(k) S_{l}(-k)$, or $S_{l}(-k) S_{l}^{T}(k)$.

Starting from RH problems of the form (1.4)-(1.5), where for convenience we have replaced $k$ by $2 k$, and using the Dressing Method, we now construct non-abelian Lax pairs.

Proposition 3.1. Let the $2 x 2$ matrix valued function $Z(x, t, k)$ satisfy the $R H$ problem (1.4)-(1.5) where the matrix $S_{l}(x, t, k)$ is replaced by $S(x, t, k)$ defined by

$$
S(x, t, k)=\left(\begin{array}{ll}
1 & e^{-i\left(2 k x+8 k^{3} t\right)} \rho_{0}(k) \\
e^{i\left(2 k x+8 k^{3} t\right)} r_{0}(k) & 1+\rho_{0}(k) r_{0}(k)
\end{array}\right),
$$

and $\rho_{0}(k), r_{0}(k) \in \mathbf{H} 1(\mathbb{R})$.

Then $Z(x, t, k)$ satisfies the following Lax pairs:

$$
Z_{x}+i k\left[\sigma_{3}, Z\right]=Q Z
$$




$$
Z_{t}+4 i k^{3}\left[\sigma_{3}, Z\right]=\left(k^{2} Q_{2}+k Q_{1}+Q_{0}\right) Z,
$$

where

$$
Q(x, t)=i\left[\sigma_{3}, \zeta_{1}\right]
$$

$$
Q_{0}=-4 \zeta_{2 x}-Q_{1} \zeta_{1}, \quad Q_{1}=-4 \zeta_{1 x}, \quad Q_{2}=4 Q,
$$

and $\zeta_{1}$ and $\zeta_{2}$ are the coefficients of the $1 / k$ and $1 / k^{2}$ terms respectively, in the large $k$ asymptotic expansion of $Z$ :

$$
Z=I_{2}+\frac{\zeta_{1}}{k}+\frac{\zeta_{2}}{k^{2}}+O\left(\frac{1}{k^{3}}\right), \quad k \rightarrow \infty .
$$

\section{Proof.}

We construct dressing operators that satisfy the same $\mathrm{RH}$ problem as $Z(x, t, k)$. We observe that the matrix $S(x, t, k)$ can be written in the form

$$
S(x, t, k)=e^{-i\left(k x+4 k^{3} t\right) \sigma_{3}}\left(\begin{array}{ll}
1 & \rho_{0}(k) \\
r_{0}(k) & 1+\rho_{0}(k) r_{0}(k)
\end{array}\right) e^{i\left(k x+4 k^{3} t\right) \sigma_{3}} .
$$

The above result implies that the expressions

$$
Z_{x}+i k \widehat{\sigma}_{3} Z, \quad Z_{t}+4 i k^{3} \hat{\sigma}_{3}
$$

satisfy the RH problem (1.4)-(1.5) with $S(x, t, k)$ defined by (3.1). Indeed, it can be verified directly that if $Z(x, t, k)$ satisfies (1.4), then

$$
\begin{aligned}
& \left(\partial_{x}+i k \widehat{\sigma}_{3}\right) Z^{+}=\left[\left(\partial_{x}+i k \widehat{\sigma}_{3}\right) Z^{-}\right] S(x, t, k), \\
& \left(\partial_{t}+4 i k^{3} \hat{\sigma}_{3}\right) Z^{+}=\left\{\left(\partial_{t}+4 i k^{3} \hat{\sigma}_{3}\right) Z^{-}\right\} S(x, t, k) .
\end{aligned}
$$

We now concentrate on the construction of the $x$-operator. We first note that if $Q(x, t)$ is an arbitrary $2 \times 2$ matrix, then

$$
Q Z^{+}=\left(Q Z^{-}\right) S \text {. }
$$

From the above it follows that the two combinations

$$
\left(\partial_{x}+i k \widehat{\sigma}_{3}\right) Z, \quad(Q Z),
$$

satisfy the the same jump condition (1.4) as the matrix $Z$. 
The basic idea of the Dressing Method is to choose the matrix $Q(x, t)$ in such a way so that the combination

$$
\left(\partial_{x}+i k \widehat{\sigma}_{3}-Q\right) Z
$$

vanishes as $k \rightarrow \infty$. Then, assuming that the Riemann-Hilbert problem (1.4)-(1.5) has a unique solution, it follows that this combination vanishes identically. The assumption that the combination (3.9) vanishes as $k \rightarrow \infty$, fixes $Q$ uniquely. Indeed, let the operator $M$ be defined by

$$
M Z=\left(\partial_{x}+i k \widehat{\sigma}_{3}-Q\right) Z
$$

Expanding $Z(x, t, k)$ in the form (3.6) and substituting this expansion into equation (3.10) we find

$$
M Z=i \widehat{\sigma}_{3} \zeta_{1}-Q+O\left(\frac{1}{k}\right), \quad k \rightarrow \infty .
$$

Thus, if $Q(x, t)$ is defined by (3.4), then $M Z \sim O\left(\frac{1}{k}\right)$, hence equation (3.2) follows.

Let $N Z$ be the $t$-operator. Since the expression $Z_{t}+4 i k^{3} \hat{\sigma}_{3} Z \sim O\left(k^{2}\right)$, we consider the following combination,

$$
N Z=\left(\partial_{t}+4 i k^{3} \widehat{\sigma}_{3}-k^{2} Q_{2}-k Q_{1}-Q_{0}\right) Z
$$

Substituting (3.6) into the above equation and demanding that $N Z \rightarrow 0$ as $k \rightarrow \infty$, we find $(3.5 \mathrm{c})$ as well as

$$
\begin{aligned}
& Q_{0}=4 i\left[\sigma_{3}, \zeta_{3}\right]-Q_{1} \zeta_{1}-4 Q \zeta_{2}, \\
& Q_{1}=4 i\left[\sigma_{3}, \zeta_{2}\right]-4 Q \zeta_{1} .
\end{aligned}
$$

The large $k$ - expansion of (3.2) implies

$$
\zeta_{1 x}+i\left[\sigma_{3}, \zeta_{2}\right]-Q \zeta_{1}=0 .
$$

and

$$
i\left[\sigma_{3}, \zeta_{3}\right]=Q \zeta_{2}-\zeta_{2 x} .
$$

Comparing equations (3.5b) and (3.15), we find (3.5a).

Also, equations (3.13) and (3.16) yield (3.5). 
In analogy with the above Proposition, starting from the matrix $\mathrm{RH}$ problem associated with the linearised Schroedinger (1.13) and sine-Gordon (2.4) equations, we find the following:

Proposition 3.2. Let the $2 x 2$ matrix valued function $Z(x, t, k)$ satisfy the $R H$ problem (1.4)-(1.5) where the matrix $S_{l}(x, t, k)$ is replaced by $S(x, t, k)$ defined by

$$
S(x, t, k)=\left(\begin{array}{ll}
1 & e^{-i\left(2 k x+4 k^{2} t\right)} \rho_{0}(k) \\
e^{i\left(2 k x+4 k^{2} t\right)} r_{0}(k) & 1+\rho_{0}(k) r_{0}(k)
\end{array}\right),
$$

and $\rho_{0}(k), r_{0}(k) \in \mathbf{H} 1(\mathbb{R})$.

Then $Z(x, t, k)$ satisfies the following Lax pair:

$$
Z_{x}+i k\left[\sigma_{3}, Z\right]=Q Z
$$

$$
Z_{t}+2 i k^{2}\left[\sigma_{3}, Z\right]=(k A+B) Z,
$$

where $Q$ is defined by (3.4),

$$
A=2 Q, \quad B=2 i\left[\sigma_{3}, \zeta_{2}(x, t)\right]-2 Q \zeta_{1}(x, t),
$$

and $\zeta_{1}$ and $\zeta_{2}$ are the coefficients of the $1 / k$, and $1 / k^{2}$ terms in (3.6).

Proposition 3.3. Let the $2 x 2$ matrix valued function $Z(x, t, k)$ satisfy the $R H$ problem (1.4)-(1.5) where the matrix $S_{l}(x, t, k)$ is replaced by $S(x, t, k)$ defined by

$$
S(x, t, k)=\left(\begin{array}{ll}
1 & e^{-i\left(2 k x+\frac{1}{2 k} t\right)} \rho_{0}(k) \\
e^{i\left(2 k x+\frac{1}{2 k} t\right)} r_{0}(k) & 1+\rho_{0}(k) r_{0}(k)
\end{array}\right),
$$

and $\rho_{0}(k), r_{0}(k) \in \mathbf{H} 1(\mathbb{R})$.

Then $Z(x, t, k)$ satisfies the following Lax pair:

$$
\begin{aligned}
& Z_{x}+i k\left[\sigma_{3}, Z\right]=Q Z, \\
& Z_{t}+\frac{i}{4 k}\left[\sigma_{3}, Z\right]=\frac{i}{4 k} \widetilde{P} Z, \quad j=1,2,
\end{aligned}
$$

where $Q$ is defined by (3.4a),

$$
\widetilde{P}=-4 i \zeta_{1 t},
$$

and $\zeta_{1}$ is the coefficient of the $1 / k$ term in (3.6).

It is also possible to consider an operator $M_{1}$, involving the same combination $\partial_{x}+i k \widehat{\sigma}_{3}$ as $M$ but with a faster decay at infinity, namely $M_{1} Z=O\left(\frac{1}{k^{2}}\right)$ as $k \rightarrow \infty$. The relevant results for the Stokes equation are obtained in the following Proposition. 
Proposition 3.4. Let the $2 x 2$ matrix valued function $Z(x, t, k)$ satisfy the $R H$ problem (1.4)-(1.5) where the matrix $S_{l}(x, t, k)$ is replaced by (3.1). Then $Z(x, t, k)$ satisfies the following Lax pair:

$$
\begin{aligned}
& Z_{x}+i k\left[\sigma_{3}, Z\right]=\frac{1}{k} \widetilde{Q} Z \\
& Z_{t}+4 i k^{3}\left[\sigma_{3}, Z\right]=\left(k \widetilde{Q}_{1}+\widetilde{Q}_{2}+\frac{1}{k} \widetilde{Q}_{3}\right) Z
\end{aligned}
$$

where

$$
\begin{aligned}
& \widetilde{Q}(x, t)=\zeta_{1 x}+i\left[\sigma_{3}, \zeta_{2}\right], \\
& \widetilde{Q}_{1}(x, t)=4 i \widehat{\sigma}_{3} \zeta_{2}(x, t), \quad \widetilde{Q}_{2}(x, t)=-\widetilde{Q}_{1}(x, t) \zeta_{1}(x, t)+4 i \widehat{\sigma}_{3} \zeta_{3}(x, t), \\
& \widetilde{Q}_{3}(x, t)=4 i \widehat{\sigma}_{3} \zeta_{4}(x, t)+\zeta_{1 t}(x, t)-\widetilde{Q}_{1}(x, t) \zeta_{2}(x, t)-\widetilde{Q}_{2}(x, t) \zeta_{1}(x, t),
\end{aligned}
$$

and $\zeta_{1}, \zeta_{2}, \zeta_{3}$, and $\zeta_{4}$ are the coefficients of the $1 / k, 1 / k^{2}, 1 / k^{3}$ and $1 / k^{4}$ terms respectively, in (3.6).

\section{Proof.}

Let the operator $M_{1}$ be defined by

$$
M_{1} Z=\left(\partial_{x}+i k \widehat{\sigma}_{3}-\frac{1}{k} \widetilde{Q}\right) Z
$$

Substituting the expansion (3.6) in the above equation we obtain

$$
M_{1} Z=i\left[\sigma_{3}, \zeta_{1}\right]+\frac{1}{k}\left\{\zeta_{1 x}+i\left[\sigma_{3}, \zeta_{2}\right]-\widetilde{Q}\right\}+O\left(\frac{1}{k^{2}}\right), \quad k \rightarrow \infty
$$

Thus, if $\widetilde{Q}$ is given by (3.27) then $Z(z, t, k)$ satisfies (3.25). Furthermore, instead of the operator $N$, we now consider the operator $N_{1}$ defined by

$$
N_{1} Z=\left(\partial_{t}+4 i k^{3} \hat{\sigma}_{3}-k \widetilde{Q}_{1}-\widetilde{Q}_{2}-\frac{1}{k} \widetilde{Q}_{3}\right) Z
$$

It is straightforward to prove that if the matrices $\widetilde{Q}_{j}, j=1, \ldots, 3$, are given by (3.28)(3.29) then $Z(z, t, k)$ satisfies (3.26). 
The linearised Schroedinger equation (1.13) possesses the Lax pair

$$
\mu_{x}+i k^{2} \mu=q(x, t), \quad \mu_{t}+i k^{4} \mu=i q_{x}+k^{2} q .
$$

Similarly to the previous cases, this Lax pair implies the following dressing operators

$$
\partial_{x}+i k^{2}-k P-R, \quad \partial_{t}+2 i k^{4}-k^{3} P_{3}-k^{2} P_{2}-k P_{1}-P_{0} .
$$

Using these operators we obtain the following result:

Proposition 3.5 Let the $2 x 2$ matrix valued function $Z(x, t, k)$ satisfy the $R H$ problem (1.4)-(1.5) where the matrix $S_{l}(x, t, k)$ is replaced by (3.17). Then $Z(x, t, k)$ satisfies the following Lax pair:

$$
\begin{aligned}
& Z_{x}+i k^{2}\left[\sigma_{3}, Z\right]=(k P+R) Z, \\
& Z_{t}+2 i k^{4}\left[\sigma_{3}, Z\right]=\left(k^{3} P_{3}-k^{2} P_{2}-k P_{1}-P_{0}\right) Z,
\end{aligned}
$$

where $Q$ is defined by (3.4),

$$
\begin{aligned}
& P=i\left[\sigma_{3}, \zeta_{1}\right], \quad R=i\left[\sigma_{3}, \zeta_{2}(x, t)\right]-P \zeta_{1}(x, t), \\
& P_{0}=2 \zeta_{1 x} \zeta_{1}-2 \zeta_{2 x}, P_{1}=-2 \zeta_{1 x}, P_{2}=2 R, P_{3}=2 P .
\end{aligned}
$$

and $\zeta_{1}$ and $\zeta_{2}$ are the coefficients of the $1 / k$, and $1 / k^{2}$ terms in (3.6).

\section{Derivation of Nonlinear Dispersive Equations}

The compatibility condition of each of the Lax pairs obtained in the previous section yields a corresponding pair of nonlinear dispersive PDEs for $q(x, t)$ and $r(x, t)$. These equations are given in the following Propositions.

Proposition 3.6. Let $q=2 i\left(\zeta_{1}\right)_{12}, r=-2 i\left(\zeta_{1}\right)_{21}, a_{1}=\left(\zeta_{1}\right)_{11}, \delta_{1}=\left(\zeta_{1}\right)_{22}, \beta_{2}=\left(\zeta_{2}\right)_{12}$ and $\gamma_{2}=\left(\zeta_{2}\right)_{21}$ where $\zeta_{1}$ and $\zeta_{2}$ is the coefficient of the $\frac{1}{k}$ and $\frac{1}{k^{2}}$ terms in the asymptotic expansion (3.6). Let the function $Z(x, t, k)$ satisfy equations (3.2) and (3.3). Then $q$, $r, a_{1}, \delta_{1}, \beta_{2}$ and $\gamma_{2}$ satisfy the following system of nonlinear dispersive equations:

$$
\begin{aligned}
& q_{t}+q_{x x x}-2\left(q^{2} r\right)_{x}-4 i q^{2} r\left(a_{1}+\delta_{1}\right)+8 q\left(-r \beta_{2}+q \gamma_{2}\right)=0, \\
& r_{t}+r_{x x x}-2\left(q r^{2}\right)_{x}+4 i q r^{2}\left(a_{1}+\delta_{1}\right)-8 r\left(-r \beta_{2}+q \gamma_{2}\right)=0 .
\end{aligned}
$$

Proof.

The diagonal part of (3.15) yields

$$
a_{1 x}=2 i \beta_{1} \gamma_{1}, \quad \delta_{1 x}=-2 i \beta_{1} \gamma_{1} .
$$


The above equation and (3.5b) yield

$$
Q_{1}=(-4)\left(\begin{array}{ll}
2 i \beta_{1} \gamma_{1} & \beta_{1 x} \\
\gamma_{1 x} & -2 i \beta_{1} \gamma_{1}
\end{array}\right) \text {. }
$$

Using (3.5a) and (4.4) we find

$$
Q_{0}=(-4)\left(\begin{array}{ll}
-2 i \beta_{1} \gamma_{1} a_{1}-\beta_{1 x} \gamma_{1}+a_{2 x} & -2 i \beta_{1}^{2} \gamma_{1}-\beta_{1 x} \delta_{1}+\beta_{2 x} \\
-\gamma_{1 x} a_{1}+2 i \beta_{1} \gamma_{1}^{2}+\gamma_{2 x} & -\gamma_{1 x} \beta_{1}+2 i \beta_{1} \gamma_{1} \delta_{1}+\delta_{2 x}
\end{array}\right) .
$$

The off diagonal and diagonal parts of (3.14) and (3.16) respectively, yield

$$
\begin{aligned}
& a_{2 x}=2 i \beta_{1} \gamma_{2}, \quad \delta_{2 x}=-2 i \beta_{2} \gamma_{1}, \\
& \beta_{1 x}=2 i \beta_{1} \delta_{1}-2 i \beta_{2}, \quad \gamma_{1 x}=-2 i \gamma_{1} a_{1}+2 i \gamma_{2} .
\end{aligned}
$$

Taking the derivative of (4.7) with respect to $x$ and substituting the resulting equation as well as the above two equations into (4.5), we find

$$
Q_{0}=\left(\begin{array}{ll}
8 i \beta_{1} \gamma_{1}\left(a_{1}+\delta_{1}\right)-8 i\left(\beta_{2} \gamma_{1}+\beta_{1} \gamma_{2}\right) & 16 i \beta_{1}^{2} \gamma_{1}-2 i \beta_{1 x x} \\
-16 i \beta_{1} \gamma_{1}^{2}+2 i \gamma_{1 x x} & -8 i \beta_{1} \gamma_{1}\left(a_{1}+\delta_{1}\right)+8 i\left(\beta_{2} \gamma_{1}+\beta_{1} \gamma_{2}\right)
\end{array}\right) .
$$

Substituting $Z=z e^{i\left(k x+4 k^{3} t\right) \sigma_{3}}$ into equations (3.2) and (3.3) and demanding that $z_{t x}=z_{x t}$ we find

$$
Q_{t}+\left[-i k \sigma_{3}+Q,-4 i k^{3} \sigma_{3}+k^{2} Q_{2}+k Q_{1}+Q_{0}\right]-k^{2} Q_{2 x}-k Q_{1 x}-Q_{0 x}=0 .
$$

The $O(1)$ terms cancel iff

$$
Q_{t}+\left[Q, Q_{0}\right]-Q_{0 x}=0 .
$$

Then, the (12) and (21) element of equation (4.10) yield equations (4.1)-(4.2).

\section{QED}

For the cases of linearised Schroedinger and sine-Gordon equations we obtain the following results:

Proposition 3.7. Let $q$ and $r$ be defined as in Proposition 3.6. Let the function $Z(x, t, k)$ satisfy equations (3.18) and (3.19).

Then $q$, and $r$ satisfy the following system of nonlinear dispersive equations:

$$
\begin{aligned}
& q_{t}-i q_{x x}+2 i q^{2} r=0, \\
& r_{t}+i r_{x x}-2 i q r^{2}=0 .
\end{aligned}
$$

\section{Proof.}


Equations (3.15) and (3.20b) imply

$$
B=-2 \zeta_{1 x} .
$$

Let $\zeta_{1}$ be defined by

$$
\zeta_{1}=\left(\begin{array}{ll}
a_{1} & \beta_{1} \\
\gamma_{1} & \delta_{1}
\end{array}\right)
$$

Equation (3.4) implies that $Q$ is given by

$$
Q=\left(\begin{array}{ll}
0 & 2 i \beta_{1} \\
-2 i \gamma_{1} & 0
\end{array}\right)
$$

Thus, equations (4.3) and (4.13) give

$$
B=\left(\begin{array}{ll}
-4 i \beta_{1} \gamma_{1} & -2 \beta_{1 x} \\
-2 \gamma_{1 x} & 4 i \beta_{1} \gamma_{1}
\end{array}\right)=-4 i \beta_{1} \gamma_{1} \sigma_{3}-i Q_{x} \sigma_{3} .
$$

Substituting $Z=z e^{i\left(k x+2 k^{2} t\right) \sigma_{3}}$ into equations (3.18) and (3.19) as well as substituting in the latter equation the matrix $B(x, t)$ by the expression (4.16) and demanding that $z_{t x}=z_{x t}$ we obtain an equation involving terms of different powers of $k$ as well as $Q$ and its derivatives. The $O(1)$ terms of this equation cancel iff

$$
Q_{t}+\left[Q,-i Q^{2} \sigma_{3}-i Q_{x} \sigma_{3}\right]+i\left(Q^{2}\right)_{x} \sigma_{3}+i Q_{x x} \sigma_{3}=0 .
$$

Taking into consideration (4.15) the (12) and (21) element of equation (4.17) yield the system (4.11)-(4.12).

\section{QED}

Proposition 3.8. Let $q, r, a_{1}$ and $\delta_{1}$ be defined as in Proposition 3.6 Let the function $Z(x, t, k)$ satisfy equations (3.22) and (3.23). Then $p, q, r$ and $s$ satisfy the following system of dispersive equations:

$$
a_{1 x t}=-\delta_{1 x t}, \quad 2 i \delta_{1 x t}=q_{t} r+r_{t} q .
$$

Proof.

Substituting the asymptotic expansion

$$
Z=Z_{0}(x, t)+k Z_{1}(x, t)+O\left(k^{2}\right), k \rightarrow 0 .
$$

into (3.23) we find

$$
\widetilde{P}=-\left[\sigma_{3}, Z_{0}\right] Z_{0}^{-1} .
$$

Letting

$$
Z_{0}=\left(\begin{array}{cc}
A_{0} & B_{0} \\
\Gamma_{0} & \Delta_{0}
\end{array}\right),
$$


equation (4.20) implies

$$
\widetilde{P}=2\left(\begin{array}{cc}
B_{0} \Gamma_{0} & -B_{0} A_{0} \\
\Gamma_{0} \Delta_{0} & -B_{0} \Gamma_{0}
\end{array}\right)
$$

Comparing equations (3.4) and (3.24) we find the following equations

$$
\begin{aligned}
& B_{0} \Gamma_{0}=-2 i a_{1 t}, \quad B_{0} \Gamma_{0}=2 i \delta_{1 t}, \\
& B_{0} A_{0}=q_{t}, \quad \Gamma_{0} \Delta_{0}=r_{t} .
\end{aligned}
$$

The matrix $\widetilde{P}$ becomes

$$
\widetilde{P}=2\left(\begin{array}{ll}
-2 i a_{1 t} & -q_{t} \\
r_{t} & -2 i \delta_{1 t}
\end{array}\right) \text {. }
$$

Substituting

$$
Z=z e^{i\left(k x+\frac{1}{4 k}\right) \sigma_{3}}
$$

into the Lax pair (3.18)-(3.23) and taking into consideration the compatibility condition $z_{t x}=z_{x t}$ we find an equation involving terms with powers of $k$ and the matrices $Q, \widetilde{Q}$. The $O\left(\frac{1}{k}\right)$ term of this equation is

$$
\widetilde{P}_{x}+[\widetilde{P}, Q]+\left[\sigma_{3}, Q\right]=0 .
$$

This equation can be rewritten as

$$
\left(\begin{array}{ll}
-2 i a_{1 t} & -q_{t} \\
r_{t} & -2 i \delta_{1 t}
\end{array}\right)_{x}+\left(\begin{array}{ll}
-q_{t} r-r_{t} q & 2 i q\left(\delta_{1 t}-a_{1 t}\right) \\
2 i r\left(a_{1 t}-\delta_{1 t}\right) & q_{t} r+r_{t} q
\end{array}\right)+\left(\begin{array}{ll}
0 & q \\
-r & 0
\end{array}\right)=0 .
$$

Therefore the (11) and (22) terms of the above equation yield equations (4.18).

Proposition 3.9. Let $\beta_{2}, \gamma_{2}, a_{1}$ and $\delta_{1}$ be defined as in Proposition 3.6. Let the function $Z(x, t, k)$ satisfy equations (3.25) and (3.26). Then $\beta_{2}, \gamma_{2}, a_{1}$ and $\delta_{1}$ satisfy the following system of nonlinear dispersive equations:

$$
\begin{aligned}
& i \beta_{2 t}+i \beta_{2 x x x}-4 \beta_{2 x}\left(a_{1 x}-\delta_{1 x}\right)-2 \beta_{2}\left(a_{1 x}-\delta_{1 x}\right)_{x}=0, \\
& i \gamma_{2 t}+i \gamma_{2 x x x}-4 \gamma_{2 x}\left(a_{1 x}-\delta_{1 x}\right)-2 \gamma_{2}\left(a_{1 x}-\delta_{1 x}\right)_{x}=0 .
\end{aligned}
$$

Proof.

Equation (3.28) implies

$$
\widetilde{Q}_{1}=4 i\left(\begin{array}{ll}
0 & 2 \beta_{2} \\
-2 \gamma_{2} & 0
\end{array}\right) .
$$


The large $k$ - expansion of (3.25) implies

$$
\begin{aligned}
& i\left[\sigma_{3}, \zeta_{1}\right]=0, \\
& \zeta_{2 x}+i\left[\sigma_{3}, \zeta_{3}\right]=\widetilde{Q} \zeta_{1}, \\
& i\left[\sigma_{3}, \zeta_{4}\right]=\widetilde{Q} \zeta_{2}-\zeta_{3 x} .
\end{aligned}
$$

Comparing equations (3.28b) and (4.32) we find:

$$
\widetilde{Q}_{2}=4 \widetilde{Q} \zeta_{1}-4 \zeta_{2 x}-\widetilde{Q}_{1} \zeta_{1} .
$$

Let $\zeta_{2}$ be defined by

$$
\zeta_{2}=\left(\begin{array}{cc}
a_{2} & \beta_{2} \\
\gamma_{2} & \delta_{2}
\end{array}\right)
$$

Then equations (3.27) and (4.31) yield

$$
\widetilde{Q}=\left(\begin{array}{ll}
a_{1 x} & 2 i \beta_{2} \\
-2 i \gamma_{2} & \delta_{1 x}
\end{array}\right) \text {. }
$$

Equations (4.30), (4.31) and (4.36) imply

$$
\widetilde{Q} \zeta_{1}=\left(\begin{array}{ll}
a_{1 x} a_{1} & 2 i \beta_{2} \delta_{1} \\
-2 i \gamma_{2} a_{1} & \delta_{1 x} \delta_{1}
\end{array}\right), \quad \widetilde{Q}_{1} \zeta_{1}=\left(\begin{array}{ll}
0 & 8 i \beta_{2} \delta_{1} \\
-8 i \gamma_{2} a_{1} & 0
\end{array}\right) .
$$

The diagonal part of (4.32) yields

$$
a_{2 x}=a_{1 x} a_{1}, \quad \delta_{2 x}=\delta_{1 x} \delta_{1} .
$$

Equation (4.34) after using (4.38) yields an expression for $\widetilde{Q}_{2}$ :

$$
\widetilde{Q}_{2}=(-4)\left(\begin{array}{ll}
0 & \beta_{2 x} \\
\gamma_{2 x} & 0
\end{array}\right) .
$$

In order to find an expression for $\widetilde{Q}_{3}$ we start from equation (3.29). Using (4.30) and (4.39) we find

$$
\widetilde{Q}_{1} \zeta_{2}=8 i\left(\begin{array}{ll}
\beta_{2} \gamma_{2} & \beta_{2} \delta_{2} \\
-\gamma_{2} \alpha_{2} & -\beta_{2} \gamma_{2}
\end{array}\right), \quad \widetilde{Q}_{2} \zeta_{1}=(-4)\left(\begin{array}{ll}
0 & \beta_{2 x} \delta_{1} \\
\gamma_{2 x} \alpha_{1} & 0
\end{array}\right)
$$

Then equation (3.29) yields

$$
\widetilde{Q}_{3}=\left(\begin{array}{ll}
-8 i \beta_{2} \gamma_{2}+a_{1 t} & -8 i \beta_{2} \delta_{2}+4 \beta_{2 x} \delta_{1}+8 i \beta_{4} \\
8 i \gamma_{2} a_{2}+4 \gamma_{2 x} a_{1}-8 i \gamma_{4} & 8 i \beta_{2} \gamma_{2}+\delta_{1 t}
\end{array}\right) .
$$

Equation (4.33) taking into consideration (4.36), yields

$$
2 i \beta_{4}=a_{1 x} \beta_{2}+2 i \beta_{2} \delta_{2}-\beta_{3 x},
$$




$$
-2 i \gamma_{4}=\delta_{1 x} \gamma_{2}-2 i \gamma_{2} a_{2}-\gamma_{3 x}
$$

Then

$$
\widetilde{Q}_{3}=\left(\begin{array}{ll}
-8 i \beta_{2} \gamma_{2}+a_{1 t} & 4 \beta_{2 x} \delta_{1}+4 a_{1 x} \beta_{2}-4 \beta_{3 x} \\
4 \gamma_{2 x} a_{1}+4 \delta_{1 x} \gamma_{2}-4 \gamma_{3 x} & 8 i \beta_{2} \gamma_{2}+\delta_{1 t}
\end{array}\right)
$$

Equation (4.32) implies

$$
\begin{aligned}
& 2 i \beta_{3}=-\beta_{2 x}+2 i \beta_{2} \delta_{1}, \\
& -2 i \gamma_{3}=-\gamma_{2 x}-2 i \gamma_{2} a_{1} .
\end{aligned}
$$

Substituting the above into (4.44), we obtain the following expression for $\widetilde{Q}_{3}$ :

$$
\widetilde{Q}_{3}=\left(\begin{array}{ll}
-8 i \beta_{2} \gamma_{2}+a_{1 t} & 4 \beta_{2}\left(a_{1 x}-\delta_{1 x}\right)-2 i \beta_{2 x x} \\
4 \gamma_{2}\left(\delta_{1 x}-a_{1 x}\right)+2 i \gamma_{2 x x} & 8 i \beta_{2} \gamma_{2}+\delta_{1 t}
\end{array}\right)
$$

Substituting $Z=z e^{i\left(k x+4 k^{3} t\right)}$ into the eigenvalue equations (3.25)-(3.26) and demanding that these equations are compatible we find

$$
\frac{1}{k} \widetilde{Q}_{t}-k \widetilde{Q}_{1 x}-\widetilde{Q}_{2 x}-\frac{1}{k} \widetilde{Q}_{3 x}+\left[-i k \sigma_{3}+\frac{1}{k} \widetilde{Q},-4 i k^{3} \sigma_{3}+k \widetilde{Q}_{1}+\widetilde{Q}_{2}+\frac{1}{k} \widetilde{Q}_{3}\right]=0
$$

The $O\left(\frac{1}{k}\right)$ term of the above equation is

$$
\widetilde{Q}_{t}+\left[\widetilde{Q}, \widetilde{Q}_{2}\right]-\widetilde{Q}_{3 x}=0
$$

The (12) and (21) terms of equation (4.49) yields the system (4.28)-(4.29).

\section{QED}

Proposition 3.10. Let $q$ and $r$ be defined as in Proposition 3.6. Let the function $Z(x, t, k)$ satisfy equations (3.35) and (3.36).

Then $q$, and $r$ satisfy the following system of nonlinear dispersive equations:

$$
\begin{aligned}
& q_{t}-i q_{x x}+i\left(q^{2} r\right)_{x}=0, \\
& r_{t}+i r_{x x}-i\left(q r^{2}\right)_{x}=0 .
\end{aligned}
$$

Proof.

Equations (3.37) yield

$$
P=\left(\begin{array}{ll}
0 & 2 i \beta_{2} \\
-2 i \gamma_{2} & 0
\end{array}\right), \quad R=\left(\begin{array}{ll}
-2 i \beta_{1} \gamma_{1} & 2 i\left(\beta_{2}-\beta_{1} \delta_{1}\right) \\
-2 i\left(\gamma_{2}-\gamma_{1} a_{1}\right) & 2 i \beta_{1} \gamma_{1}
\end{array}\right)
$$


Also, equation (3.38c) implies

$$
\widetilde{P_{0}}=\left(\begin{array}{cc}
a_{1 x} a_{1}+\beta_{1 x} \gamma_{1}-a_{2 x} & a_{1 x} \beta_{1}+\beta_{1 x} \delta_{1}-\beta_{2 x} \\
\delta_{1 x} \gamma_{1}+\gamma_{1 x} a_{1}-\gamma_{2 x} & \delta_{1 x} \delta_{1}+\gamma_{1 x} \beta_{1}-\delta_{2 x}
\end{array}\right) .
$$

Following precisely analogous steps to the previous cases we obtain the following equations

$$
\begin{aligned}
& i\left[\sigma_{3}, \widetilde{P_{0}}\right]=-2 B_{x}+\left[A,-2 \zeta_{1 x}\right], \\
& P_{t}+2 \zeta_{1 x x}+2\left[P, \widetilde{P_{0}}\right]+\left[R,-\zeta_{1 x}\right]=0 .
\end{aligned}
$$

The first of the above equations implies

$$
a_{1}=\delta_{1} .
$$

Then, the (12) and (21) terms of (4.55) yield the system (4.50)-(4.51).

\section{QED}

\section{Nonlinear Integrable Equations}

Considering appropriate reductions of the above systems of nonlinear dispersive equations yields single nonlinear integrable versions of the linear PDEs considered in Proposition 2.1 .

Modified KdV. The system (4.1)-(4.2) admits the reduction $r=-q$. Then, equations (4.3) and (4.7) yield $a_{1}=-\delta_{1}$ and $\beta_{2}=-\gamma_{2}$ and this system reduces to the modified KdV equation (1.11).

Nonlinear Schroedinger. The reduction $r=\lambda \bar{q}, \lambda= \pm 1$, implies that the system (4.11)-(4.12) reduces to the NLS equation (1.15) as well as to the complex conjugate of this equation.

Derivative NLS. The reduction $r=\lambda \bar{q}, \lambda= \pm 1$, reduces the system (4.50)-(4.51) to derivative NLS (1.16) and its complex conjugate.

sine-Gordon. Letting

$$
q=r=\frac{f_{x}}{2}
$$

in equation (4.23) it follows that

$$
A_{0} B_{0}-\Gamma_{0} \Delta_{0}=0 .
$$

The choice

$$
A_{0}=\Delta_{0}=\cos \frac{f}{2}, \quad B_{0}=\Gamma_{0}=\sin \frac{f}{2},
$$

satisfies equation (5.2). Then, equation (4.22) yields

$$
2 i a_{1 t}=-2 i \delta_{1 t}=-\sin ^{2} \frac{f}{2}
$$


Using (5.1) and (5.4), equations (4.18) reduce to the nonlinear sine-Gordon equation:

$$
f_{x t}=\sin f .
$$

KdV. Substituting the expansion,

$$
Z=Z_{0}+k Z_{1}+O\left(k^{2}\right), \quad k \rightarrow 0,
$$

into (3.25) we find

$$
\widetilde{Q} Z_{0}=0 .
$$

The above equation implies that one should restrict the form of either $\widetilde{Q}$ or $\widetilde{Z}$. Restricting $Z$ can lead to inconsistencies, therefore we require the matrix $\widetilde{Q}$ to be singular, namely $\operatorname{det} \widetilde{Q}=0$, or equivalently

$$
a_{1 x} \delta_{1 x}=4 \beta_{2} \gamma_{2} .
$$

The $O\left(\frac{1}{k^{2}}\right)$ term of equation (4.48) yields

$$
\left[\widetilde{Q}, \widetilde{Q}_{3}\right]=0 .
$$

A simple algebraic manipulation of this equation implies

$$
\beta_{2}=\gamma_{2} .
$$

Then the reduction

$$
a_{1 x}=-\delta_{1 x}=\frac{q}{2 i},
$$

and equation (5.8) imply that the system (4.28)-(4.29) reduces to the Korteweg-de-Vries equation (1.12).

\section{Conclusions}

The modelling of a variety of important physical phenomena requires the formulation of nonlinear partial differential equations (PDEs). There exists a particular class of such equations which are called integrable, and which possess two distinctive characteristics: first, they appear in many areas of mathematics and physics and secondly, they can be investigated analytically using the inverse scattering method. Regarding the first characteristic we note that physical applications of integrable equations include ion-acoustic, electromagnetic, electrostatic, ionospheric, and water waves, stimulated Raman scattering, biology, relativity, and quantum field theory (see for example [1]). This reflects the fact these equations express a certain physical coherence, which is present in a variety of physical phenomena. Calogero and Eckhaus have shown [3],[4] that the ubiquitous occurence of integrable PDEs is due to the fact that they can be obtained from very large classes of nonlinear evolution equations through a limiting procedure involving rescalings and an appropriate asymptotic expansion. Regarding the inverse scattering method we note that 
for equations in $1+1$ dimensions this method is based on the spectral analysis of an eigenvalue equation which in turn requires the use of the Riemann-Hilbert $(\mathrm{RH})$ formalism. The generalisation of this formalism needed for the investigation of initial-boundary value problems, for the nonlinear Schroedinger, KdV, modified KdV and sine-Gordon equations has been developed in [8], [14], [9], [12], [11] and [13].

The Dressing Method is a general method for the derivation of both nonlinear integrable PDEs and large classes of their solutions. This method was introduced by Zakharov and Shabat in [17]. The first version of this method was based on a Gelfand-Levitan-Marchenko (GLM) type equation, while its second version [18] was based on the RH formalism. In the second version, one first postulates a matrix $\mathrm{RH}$ problem and then constructs appropriate dressing operators.

We present an approach for the systematic derivation of nonlinear integrable partial differential equations in 1+1 dimensions. Starting from model linear equations and using the Dressing Method, we first derive systems of nonlinear dispersive PDEs. Then, considering appropriate reductions of these systems we obtain nonlinear integrable equations.

We show how one can construct in an algorithmic way matrix Riemann-Hilbert (RH) problems appropriate for the Dressing Method as opposed to postulating them ad hoc. Furthermore, we introduce two mechanisms for the construction of the relevant dressing operators. Each of these mechanisms yields different nonlinearities. In particular, the first mechanism uses operators with the same dispersive part, but with different decay at infinity. As an illustrative example of this mechanism, we start with the Stokes equation and obtain the $\mathrm{KdV}$ and modified $\mathrm{KdV}$ equations. The second mechanism uses pairs of operators corresponding to different Lax pairs of the same linear equation. We demonstrate this mechanism using the example of the linearised Schroedinger equation from which we derive the NLS and Derivative NLS equations.

Our approach can be applied to a large class of linear equations of physical significance, including equations of the form (2.7). It is expected that some of the resulting nonlinear integrable equations will also be of physical significance.

A preliminary version of this work appeared in [10].

Acknowledgments. The author is grateful to Professor A $S$ Fokas for suggesting this investigation and elucidating discussions as well as to Dr B Pelloni for useful comments and suggestions.

\section{References}

[1] Ablowitz M J and Segur H, Solitons and the Inverse Scattering Transform, SIAM Stud. Appl. Math. Philadelphia (1981)

[2] Ablowitz M J and Fokas A S, Complex Variables: Introduction and Applications, 2nd edition, Cambridge University Press (2003)

[3] Calogero F, in What is Integrability, V.E. Zakharov (ed.), Springer (1992)

[4] Calogero F and Eckhaus W, Nonlinear Evolution Equations, Rescalings, Model PDEs and Integrability, Part I: Inv. Probl. 3 (1987) 229; Part II: Inv. Probl. 4 (1988) 11

[5] FoKas A S, A Unified Transform Method for Solving Linear and Certain Nonlinear PDEs, Proc. R. Soc. A, 453 (1997) 1411 
[6] Fokas A S, A New Transform Method for Evolution PDEs, IMA J. Appl. Math. 67 (2002) 559

[7] Fokas A S and Gelfand I M, Integrability of Linear and Nonlinear Evolution Equations and the Associated Nonlinear Fourier Transforms, Lett. Math. Phys., 32 (1994) 189

[8] Fokas A S and Its A R, An Initial-Boundary Value Problem for the sine-Gordon equation in laboratory coordinates, Theor. Math. Phys. 92 (1992) 387

[9] Fokas A S and Its A R, An Initial-Boundary Value Problem for the KdV equation, Maths Comput. Simul., 37 (1994) 293

[10] Pinotsis D A, The Dbar formalism, Quaternions and Applications, PhD Thesis, University of Cambridge (2006)

[11] Boutet de Monvel A, Fokas A S and Shepelsky D, The Modified KdV Equation on the Half-Line, J. of the Inst. of Math. Jussieu 3 (2004) 139

[12] Treharne P and Fokas A S, The Generalised Dirichlet to Neumann Map for the KdV on the Half-Line (preprint)

[13] Fokas A S and Its A R, The linearisation of the Initial-Boundary Value Problem for the NLS equation, SIAM J Math Analysis, 27 (1996) 738

[14] Fokas A S, Its A R and Sung LY, The Nonlinear Schroedinger Equation on the Half-Line, Nonlinearity 18 (2005) 1771

[15] FokAs A S and Zakharov V E, The Dressing Method and Nonlocal Riemann-Hilbert problems, J Nonlinear Sci, 2 (1992) 109

[16] Lax P D, Comm. Pur. Appl. Math., 21 (1968) 467

[17] Zakharov V E and Shabat A B, Integration of nonlinear equations of mathematical physics by the method of the inverse scattering problem, Funct. Anal. Appl. 8 (1974) 226

[18] Zakharov V E and Shabat A B, Integration of nonlinear equations of mathematical physics by the method of the inverse scattering problem, Part II, Funct. Anal. Appl. 13 (1979) 166 